\title{
Effect of vitamin $A$ and $Z n$ supplementation on indices of vitamin A status, haemoglobin level and defecation of children with persistent diarrhea
}

\author{
Yuting Wang, ${ }^{1,2}$ Yuan Gao, ${ }^{2,3}$ Quanbo Liu, ${ }^{1,2}$ Xue Zhan, ${ }^{1,2}$ Zhongyue Li, ${ }^{1,2}$ Huajian Hu, ${ }^{1,2}$ Tingyu Li ${ }^{2,3}$ and Jie Chen ${ }^{2,3, *}$ \\ ${ }^{1}$ Department of Gastroenterology and ${ }^{3}$ Children's Nutrition Research Center, Children's Hospital of Chongqing Medical University, \\ Zhongshan Second Road, Yuzhong District, Chongqing 400014, China \\ ${ }^{2}$ Ministry of Education Key Laboratory of Child Development and Disorders, Key Laboratory of Pediatrics in Chongqing, \\ Zhongshan Second Road, Yuzhong District, Chongqing 400014, China
}

\begin{abstract}
To investigate the effect of vitamin $A$ and $Z n$ supplementation on vitamin A status, haemoglobin level and defecation of children with persistent diarrhea, a total of 160 paediatric patients were randomly assigned to one of four intervention groups: daily supplementation of 1,500 IU VA for 14 days; daily $Z$ n supplementation for 14 days; daily supplementation with both VA and $\mathrm{Zn}$ for 14 days; no supplementation. One hundred twenty-seven children with persistent diarrhea finished intervention ( 33 were lost to follow-up). Among the 127 children, 41 (32.28\%) had anaemia, $104(81.89 \%)$ had a VA deficiency and $38(29.92 \%)$ had an iron insufficiency. Supplementation with VA or VA $+Z n$ enhanced the serum VA levels and ameliorated anaemia. Supplementation with $\mathrm{Zn}$ and $V A+Z n$ for 5 days significantly improved defecation, where the VA + Zn treatment resulted in superior outcomes. After 14 days of intervention, the total effectiveness rates were $93.94 \%, 96.77 \%$ and $96.67 \%$ in the three groups, significantly greater than that of the non-supplementation group $(72.73 \%)$. These results indicate that single VA or concurrent VA + Zn supplementation can improve vitamin A status, haemoglobin level and defecation. However, concurrent VA $+\mathrm{Zn}$ supplementation is the optimal option and can shorten the duration of persistent diarrhea and markedly improve nutritional status. (www.clinicaltrials.gov registration number: ChiCTR-IOR-14005498)
\end{abstract}

Key Words: vitamin A, Zn, treatment efficacy of persistent diarrhea, intervention, anaemia

$\mathrm{D}$ iarrhea remains one of the top seven diseases that threaten the lives of children under 5 years old in developing countries. Based on the course of disease, diarrhea can be classified as acute or persistent. Persistent diarrhea, also known as chronic diarrhea, refers to diarrhea persisting for more than 2 weeks. ${ }^{(1)}$ Since the beginning of this century, our understanding of the pathogenesis of diarrhea has increased significantly, and treatments for this disease have greatly improved. However, in 2008, diarrheal disease accounted for 1,336 million of the estimated 8,795 million deaths $(15 \%)$ in children younger than 5 years of age around the world. ${ }^{(2)}$ Persistent diarrhea tends to cause malnutrition and growth retardation because the children are trapped in a vicious cycle of "diarrhea-malnutrition-diarrhea" and correspondingly suffer severe health consequences. These consequences impose heavy economic and social burdens on society and families. Hence, a crucial clinical task is improving the malnutrition status of children with persistent diarrhea and decreasing their disease duration.
Vitamin A deficiency (VAD) is one of the three global micronutrient deficiency diseases reported by UNICEF and WHO and is also a leading risk factor for severe infective diseases and death risk in children. Moderate VAD is endemic in China. Our group conducted a preliminary population survey. In 2008, we reported that the incidence of marginal VAD among pre-schoolers in a Chongqing suburb was approximately $30 \%$; however, the rate was higher in rural and poor mountainous areas. ${ }^{(3)}$ Qiu et al. ${ }^{(4)}$ reported that the rate of VA deficiency in children with diarrhea was 2.37fold greater than that in normal children. Furthermore, increased episodes of diarrhea were correlated with higher VA deficiencies. A survey in India indicated that VA deficiency is an independent risk factor for persistent diarrhea. ${ }^{(5)}$ In light of these findings, we investigated whether vitamin A supplementation could ameliorate the malnutrition status of children with persistent diarrhea and improve treatment outcomes. In this study, children with persistent diarrhea were administered with VA auxiliary treatment, with routine $\mathrm{Zn}$ intervention as a positive control. Improvements in the nutritional status of children with persistent diarrhea were monitored, and treatment outcomes were evaluated.

\section{Study Subjects and Methods}

Study subjects. Paediatric patients with persistent diarrhea admitted to the Department of Gastroenterology, Children's Hospital of Chongqing Medical University between April 2013 and February 2014 were recruited in this study. All study subjects were children that had suffered from diarrhea for over 14 days who met the diagnostic criteria for persistent diarrhea. ${ }^{(6)}$ The patients had no severe complications of the heart, liver, kidney or hematopoietic systems and were HIV negative. Using a computergenerated random number table, in which each number corresponded to a study serial number assigned to each paediatric patient, the children were randomly allocated into four groups: VA supplementation group (group A), Zn supplementation group (group B), VA + Zn supplementation group (group C) and no supplementation group (group D). Informed consent was obtained from each patient's guardian. This study was approved by the Clinical Ethics Committee of the Children's Hospital of Chongqing Medical University (approval number 008/2013).

*To whom correspondence should be addressed. E-mail: jchen010@cqmu.edu.cn 
Sample size calculation. We estimated that after VA intervention, the minimal value of the $\mathrm{Hb}$ increment, $\delta$, was $5 \mathrm{~g} / \mathrm{L}(5 \mathrm{~g} / \mathrm{L}$ was the observed value in our previous study) and that the standard errors for the group were the following: $\mathrm{s}=5 \mathrm{~g} / \mathrm{L}, \alpha=0.05$, and $\beta=0.01$. Using the following equation, we estimated the follow-up loss rate to be $20 \%$; The number of each group was calculated as follows: $n=[(\mathrm{U} \alpha+\mathrm{U} \beta) \times \mathrm{s} / \delta]^{2}=[(1.645+1.282) \times$ $10 / 5]^{2}=34$ (individuals). Thus, the total number of this study was $\mathrm{N}=n \div(1-20 \%) \times 4=34 \div(1-20 \%) \times 4=160$ (children).

One hundred sixty paediatric patients with persistent diarrhea were randomly assigned to one of the four intervention groups: group A (daily supplementation of 1,500 IU VA for 14 days); group B (daily $\mathrm{Zn}$ supplementation for 14 days); group $\mathrm{C}$ (daily supplementation with both VA and $\mathrm{Zn}$ for 14 days); group D (no supplementation).

Study protocol. In addition to their routine treatment, the study subjects were also treated with daily supplementation of $1,500 \mathrm{IU} \mathrm{VA}$ and/or daily supplementation of $\mathrm{Zn}(10 \mathrm{mg} /$ day for children $<6$ months old and $20 \mathrm{mg}$ /day for children $\geq 6$ months old) for 14 days. During the children's hospitalisation, dedicated nurses were responsible for providing them with medications. The stool and disease conditions of each patient were recorded by a specialised resident. If the intervention course was less than 14 days at the time of discharge, the mother or another guardian of the child was responsible for the remaining period of drug administration, which was monitored by a nurse via telephone follow-ups to ensure that the full 14-day course of VA and/or $\mathrm{Zn}$ supplementation was fulfilled. On day 15 , when the child returned for outpatient follow-up, his/her mother or guardian was questioned by a specialised physician with respect to the child's stool and other information.

Data collection. Upon admission, the background information (gender, age in months) and clinical manifestations (stool frequency, properties and systemic symptoms) of each child with persistent diarrhea were documented in detail. Prior to intervention, venous blood was drawn to examine serum biochemical parameters, including levels of VA, Zn, iron, haemoglobin and albumin. In addition, stool was collected for faecal smear tests. After 14 days of intervention, the serum VA level and haemoglobin levels were re-examined. All blood specimens were analysed in the clinical laboratory at our hospital (ISO 15189 approval, laboratory certification CNAS MT0038).

Diagnostic criteria. A child was diagnosed as VA normal (VAN) if his/her serum retinol concentration was $\geq 0.7 \mu \mathrm{M}$ or as VA deficient (VAD) if his/her serum retinol concentration was $<0.7 \mu \mathrm{M}$. In addition, serum $\mathrm{Zn}<10.7 \mu \mathrm{M}$ was defined as $\mathrm{Zn}$ deficiency. The diagnostic criteria of anaemia were as follows: $\mathrm{Hb}<90 \mathrm{~g} / \mathrm{L}$ for children 1-4 months old, $<100 \mathrm{~g} / \mathrm{L}$ for children 4 6 months old, $<110 \mathrm{~g} / \mathrm{L}$ for children 6 months old to 6 years old and $<120 \mathrm{~g} / \mathrm{L}$ for children 6 years old to 14 years old. Albumin (Alb) levels $\geq 35 \mathrm{~g} / \mathrm{L}$ were defined as Alb normal, and Alb levels $<35 \mathrm{~g} / \mathrm{L}$ were defined as hypoalbuminemia. The normal value of serum iron is $10.7-34.0 \mu \mathrm{M}$. If a child's body weight was less than the mean minus the SD of the reference group (same age, same gender), he/she was considered a low body weight patient.

The evaluation criteria of the efficacy of persistent diarrhea treatment were as follows: i) significantly effective treatment: stool traits and frequency returned to normal, and systemic symptoms resolved; ii) effective treatment: stool traits and frequency and systemic symptoms clearly improved; and iii) ineffective treatment: stool traits and systemic symptoms exhibited no improvement or worsened. ${ }^{(7)}$

The assessment of intestinal microbiota dysbiosis was based on the Fecal smear atlas for intestinal microbiota as follows: i) normal: the proportion of normal microbes/total bacterial count and the proportions of different bacterial types were normal, and the proportion of bacilli/cocci was $3: 1$ or higher; ii) degree I dysbiosis: the total bacterial count was at or slightly below the lower end of the normal range, the level of $\mathrm{Gram}^{+}$bacilli was low-normal, the level of Gram- cocci was normal-high, and the proportion of bacilli/cocci was typically $1: 1-2: 1$; iii) degree II dysbiosis: the total bacterial count was clearly reduced or exhibited no apparent change, the level of $\mathrm{Gram}^{+}$bacilli was significantly decreased, the proportion of $\mathrm{Gram}^{-}$cocci was clearly increased and reached as high as $90 \%$, resulting in a reversed proportion of bacilli/cocci, and yeast species or Clostridium species were apparently increased; and iv) degree IV dysbiosis: the total bacterial count was significantly reduced and the composition of the original faecal microbiota was significantly altered with one predominant bacterial or fungal species. ${ }^{(8)}$

Statistical methods. IBM SPSS Statistics 21.0 (International Business Machines Corporation, Newyork, NY) was used for statistical analyses. Measurement data were expressed as mean $\pm \mathrm{SD}$. Inter-group comparisons were made using the $t$ test, ANOVA or Kruskal-Wallis Test. Count data were examined via $\mathrm{R} \times \mathrm{C} \chi^{2}$ test. Linear correlation analysis was performed between different variables. A value of $p<0.05$ was considered statistically significant.

\section{Results}

General information. A total of 160 paediatric patients with persistent diarrhea were examined in this study. Thirty-three children were lost to follow-up due to a change in the guardian's contact number or relocation for work, yielding a follow-up loss rate of $20.6 \%$ (Fig. 1). The remaining 127 children with persistent diarrhea and their families completed the entire trial, which included 81 males and 46 females $(\mathrm{M}: \mathrm{F}=1.76: 1)$. The average age of the 127 children was $8.43 \pm 7.45$ months old. As shown in Table 1, the sample included 113 children less than 1 years old, representing $89 \%$ of the total patient group (31 subjects less than 3 months old, 37 subjects $4-6$ months old and 45 subjects 7-12 months old) as well as 8 children 1-3 years old and 6 children older than 3 years old.

Causes of diarrhea. The 127 paediatric patients with persistent diarrhea were clinically diagnosed as the following: 53 cases $(42 \%)$ of a food allergic gastrointestinal disease (FAG), 22 children with secondary lactose intolerance $(17 \%), 19$ children with antibiotic-associated diarrhea (AAD) (15\%), 6 children with an enteric infection (5\%) and 15 cases with unknown causes (11\%). Additionally, the other patients had a primary immunodeficiency disease, Bartter syndrome, renal tubule acidosis (RTA), primary enteric lymphangiectasis, Ulcerative colitis, graft versus host disease (GVHD), congenital adrenal cortical dysfunction, etc.

Furthermore, the faeces analysis of the pathological bacteria showed that among the 15 cases of FAG with infection, 9 cases suffered from Rotavirus enteritis, 3 cases were infected by Escherichia coli, and in the other 3 cases, the exact pathological bacteria were not identified. We cultured the stool of 6 children with only an enteric infection and found 2 cases of Salmonella typhimurium, one case with Salmonella paratyphi $C, 2$ cases with Escherichia coli and 1 case with a Pseudomonas aeruginosa infection.

Serum biochemical test. Among the 127 children with persistent diarrhea, there were 39 subjects with low body weight, representing $30.71 \%$ of the total patient sample; the incidence of low body weight was significantly higher in our patient population than China's national average in $2002(p<0.05) .^{(9,10)}$ The average $\mathrm{Hb}$ of the sample was $108.52 \pm 15.27 \mathrm{~g} / \mathrm{L}$, including 41 cases of anaemia $(32.28 \%)$; the incidence of anaemia was also significantly higher than the national average $(p<0.05) .{ }^{(11)}$ The average albumin level was $32.37 \pm 5.42 \mathrm{~g} / \mathrm{L}$, and there were 76 patients with low albumin levels $(59.84 \%)$. The average VA level was $0.75 \pm 0.32 \mu \mathrm{M}$, and there were 104 cases of VA deficiency $(81.89 \%)$, which was significantly higher than the national average $(p<0.05) ;{ }^{(12)}$ however, no cases exhibited the classical 


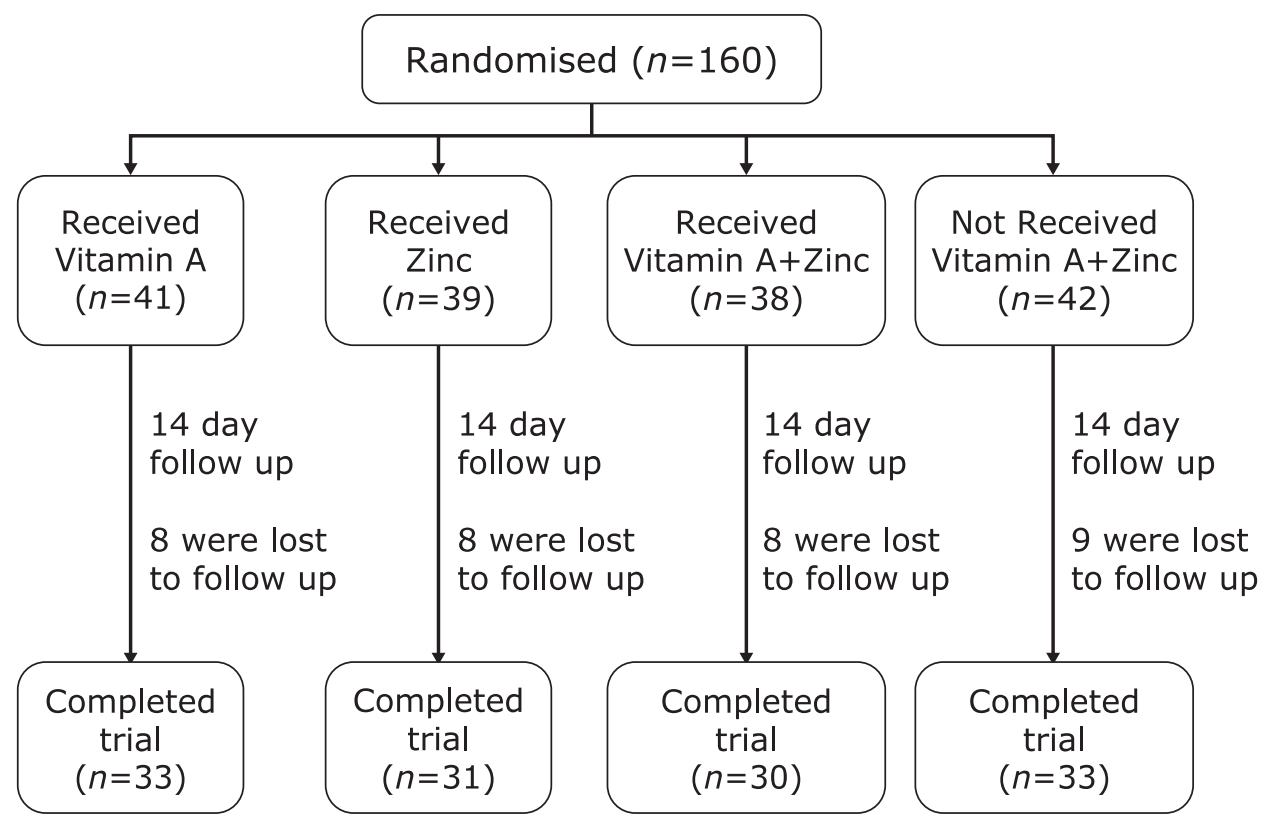

Fig. 1. Flow chart.

Table 1. Analysis of 127 children with persistent diarrhea

\begin{tabular}{lcc}
\hline & & Number $(n)$ \\
\hline Gender & Male & 81 \\
& Female & 46 \\
\hline Age & $\leq 3$ months & 31 \\
& $4-6$ months & 37 \\
& $7-12$ months & 45 \\
& $1-3$ years & 8 \\
& $\geq 3$ years & 6 \\
\hline Weight-for-age & normal & 88 \\
& low body weight & 39 \\
\hline Hemoglobin $(\mathrm{Hb})$ & normal & 86 \\
& anemia & 41 \\
\hline Albumin level & normal & 51 \\
& Hypoproteinemia & 76 \\
\hline Vitamin A level & normal & 23 \\
& VA deficiency & 104 \\
\hline Zinc level & normal & 106 \\
& Zinc insufficient & 21 \\
\hline Serum iron level & normal & 89 \\
& iron insufficient & 38
\end{tabular}

symptom of VAD. The average serum Zn level was $12.99 \pm$ $3.35 \mu \mathrm{M}$, which was markedly lower than the national average $(p<0.05){ }^{(13)}$ There were 21 cases of serum $\mathrm{Zn}$ deficiency $(16.54 \%)$. The average serum iron level was $13.51 \pm 6.36 \mu \mathrm{M}$, and there were 38 cases with below normal levels of serum iron $(29.92 \%)$ (Table 1).
Faecal microbiota analysis. The faecal smear examination for the 127 children with persistent diarrhea revealed that 120 subjects $(94.49 \%)$ exhibited various levels of microbiota dysbiosis. In addition, $78.74 \%(100 / 127)$ of the children exhibited both VA deficiency and dysbiosis. Spearman correlation analysis revealed that the VA nutritional level was correlated with a microbiota imbalance (correlation coefficient $0.212, p<0.05$ ). Based on the differential VA nutritional levels, we divided the 127 subjects into two groups: VAN and VAD. The faecal smear results of these two groups are presented in Table 2 . The 23 children in the VAN group exhibited a dysbiosis rate of $86.96 \%(20 / 23)$, and the majority of cases were degrees I and II dysbiosis (30.4\% and $47.8 \%$ ). In comparison, the dysbiosis rate in the VAD group reached $96.15 \%$ and included mostly degree II dysbiosis (74.04\%). Degrees II and III dysbiosis were grouped together as mediumsevere microbiota imbalance; thus, there were 84 children $(80.77 \%)$ in the VAD group, and the incidence of microbiota imbalance was significantly higher than that of the VAN group $(56.50 \%)$. The differences between the two groups were statistically significant $\left(\chi^{2}=6.14, p<0.01\right)$

Analysis of random grouping. The 127 children with persistent diarrhea were randomly divided into four groups to perform the study. There were 33, 31, 30 and 33 children in groups $\mathrm{A}-\mathrm{D}$, respectively, and the $\mathrm{M} / \mathrm{F}$ ratios and mean ages are presented in Table 3. Statistical analysis revealed that there were no significant differences with respect to gender and age among the individual groups $(p>0.05)$.

The average body weight, haemoglobin level, albumin level, serum VA level and $\mathrm{Zn}$ and Fe levels of the four groups before the interventions are summarised in Table 4. Our analysis indicated that prior to the intervention, the nutritional status, serum VA

Table 2. Analysis of intestinal flora smear between the VAN and VAD groups

\begin{tabular}{|c|c|c|c|c|c|}
\hline \multirow{2}{*}{ Group } & \multirow{2}{*}{ Normal $n(\%)$} & \multicolumn{3}{|c|}{ Dysbacteriosis } & \multirow{2}{*}{$\begin{array}{c}\text { Serious dysbacteriosis } \\
\text { a = II + III (\%) }\end{array}$} \\
\hline & & Degrees I $n(\%)$ & Degrees II $n(\%)$ & Degrees III $n(\%)$ & \\
\hline VAN & $3(13.0)$ & $7.0(30.40)$ & $11.0(47.80)$ & $2.0(8.70)$ & $13.0(56.50)^{*}$ \\
\hline VAD & $4(3.85)$ & $16.0(15.38)$ & 77.0 (74.03) & $7.0(6.73)$ & $84.0(80.77)$ \\
\hline
\end{tabular}

${ }^{*} \chi^{2}=6.14$, vs the VAD group, $p<0.05$. 
Table 3. The gender composition and average age among the four groups ${ }^{\dagger}$

\begin{tabular}{|c|c|c|c|c|}
\hline \multirow{2}{*}{ Group } & \multirow{2}{*}{ Number } & \multicolumn{2}{|c|}{ Gender } & \multirow{2}{*}{ Age (months) } \\
\hline & & Male & Female & \\
\hline A & 33 & 21 & 12 & $8.72 \pm 7.29$ \\
\hline B & 31 & 20 & 11 & $8.50 \pm 7.38$ \\
\hline C & 30 & 18 & 12 & $8.21 \pm 7.01$ \\
\hline $\mathrm{D}$ & 33 & 22 & 11 & $8.86 \pm 8.34$ \\
\hline Total & 127 & 81 & 46 & $8.43 \pm 7.45$ \\
\hline
\end{tabular}

Results are expressed as mean \pm SD. ${ }^{\dagger}$ There are no significant differences among the groups, $p>0.05$.

Table 4. Comparison of nutritional status between groups $^{\dagger}$

\begin{tabular}{cccccccc}
\hline Group & Number & Wt $(\mathrm{kg})$ & $\mathrm{Hb}(\mathrm{g} / \mathrm{L})$ & Alb $(\mathrm{g} / \mathrm{L})$ & $\mathrm{Zn}(\mathrm{mM})$ & $\mathrm{Fe}(\mu \mathrm{M})$ & $\mathrm{VA}(\mathrm{mM})$ \\
\hline A & 33 & $8.50 \pm 1.94$ & $109.12 \pm 11.23$ & $33.0 \pm 3.89$ & $12.64 \pm 3.30$ & $13.13 \pm 5.05$ & $0.73 \pm 0.30$ \\
B & 31 & $7.22 \pm 1.95$ & $112.52 \pm 11.85$ & $34.14 \pm 4.0$ & $13.94 \pm 3.91$ & $13.13 \pm 7.97$ & $0.79 \pm 0.33$ \\
C & 30 & $7.26 \pm 1.76$ & $111.86 \pm 15.17$ & $33.09 \pm 5.29$ & $12.83 \pm 2.95$ & $13.12 \pm 6.25$ & $0.73 \pm 0.42$ \\
D & 33 & $7.06 \pm 2.53$ & $101.32 \pm 19.42$ & $29.58 \pm 6.92$ & $12.59 \pm 3.86$ & $14.59 \pm 6.10$ & $0.74 \pm 0.26$ \\
\hline
\end{tabular}

Results are expressed as mean \pm SD. 'There are no significant differences among the groups, $p>0.05$.

Table 5. The serum VA levels among the four groups after 14 days intervention

\begin{tabular}{ccccc}
\hline Group & Number & $\begin{array}{c}\text { Mean of VA level } \\
(\mathrm{mM})\end{array}$ & $\begin{array}{c}\text { Mean of VA level after 14 days } \\
\text { intervention }(\mathrm{mM})\end{array}$ & $\begin{array}{c}\text { Mean of average increase } \\
(\mathrm{mM})\end{array}$ \\
\hline A & 33 & $0.73 \pm 0.30$ & $0.98 \pm 0.40$ & $0.24 \pm 0.10^{*}$ \\
B & 31 & $0.79 \pm 0.33$ & $0.85 \pm 0.38$ & $0.06 \pm 0.05$ \\
C & 30 & $0.73 \pm 0.42$ & $1.01 \pm 0.51$ & $0.28 \pm 0.09^{\dagger}$ \\
D & 33 & $0.74 \pm 0.26$ & $0.79 \pm 0.30$ & $0.05 \pm 0.03$ \\
\hline
\end{tabular}

Results are expressed as mean $\pm S D .{ }^{*}$ Compared with group $B, p<0.05 .{ }^{+}$Compared with group $D, p<0.01$.

Table 6. The $\mathrm{Hb}$ levels among the four groups after 14 days intervention

\begin{tabular}{ccccc}
\hline Group & Number & $\begin{array}{c}\text { Mean of Hb level } \\
(\mathrm{g} / \mathrm{L})\end{array}$ & $\begin{array}{c}\text { Mean of Hb level after 14 days } \\
\text { intervention }(\mathrm{g} / \mathrm{L})\end{array}$ & $\begin{array}{c}\text { Mean of average increase } \\
(\mathrm{g} / \mathrm{L})\end{array}$ \\
\hline A & 33 & $109.12 \pm 11.23$ & $114.22 \pm 13.47$ & $5.11 \pm 2.23^{*}$ \\
B & 31 & $112.52 \pm 11.85$ & $114.52 \pm 13.55$ & $2.43 \pm 1.70$ \\
C & 30 & $111.86 \pm 15.17$ & $119.28 \pm 19.85$ & $7.43 \pm 4.67^{\dagger}$ \\
D & 33 & $101.32 \pm 19.42$ & $103.65 \pm 20.52$ & $2.23 \pm 1.09$ \\
\hline
\end{tabular}

Results are expressed as mean $\pm S D$. * ${ }^{*}$ Compared with group $B, p<0.05 .{ }^{+}$Compared with group $D, p<0.01$.

level, serum $\mathrm{Zn}$ and Fe levels did not differ among the four groups $(p>0.05)$, which laid the basis for a subsequent study on auxiliary treatment using VA and Zn.

\section{Analyses of post-intervention results}

Post-intervention changes in $\mathrm{VA}, \mathrm{Hb}$ and serum iron levels.

Table 5 presents VA levels of the patients with persistent diarrhea before and after the intervention. After the intervention, groups $\mathrm{A}$ and $\mathrm{C}$ exhibited increases of $0.24 \pm 0.10 \mu \mathrm{M}$ and $0.28 \pm 0.09 \mu \mathrm{M}$ in the patients' VA levels, respectively. Chi-Square analysis revealed that the VA levels differed significantly from that of the groups $\mathrm{B}$ and $\mathrm{D}(p<0.05)$. There was no significant difference between group A (VA supplementation) and group $\mathrm{C}(\mathrm{VA}+\mathrm{Zn}$ supplementation), although the VA elevation in group $\mathrm{C}$ was more pronounced. In addition, the $\mathrm{Hb}$ and VA levels in the four groups before and after treatment exhibited similar trends (Table 6). After 14 days of intervention, the $\mathrm{Hb}$ level in all of the groups improved to varying degrees, with groups $\mathrm{A}$ and $\mathrm{C}$ exhibiting the most significant improvements $(p<0.05)$. The aforementioned results indicate that VA or VA $+\mathrm{Zn}$ supplementation, particularly the latter, can improve VA levels and ameliorate anaemia in paediatric patients with persistent diarrhea.

The changes of serum iron following intervention are shown in Table 7. After the intervention, groups $\mathrm{A}$ and $\mathrm{C}$ displayed decreases of $1.11 \pm 1.14 \mu \mathrm{M}$ and $0.30 \pm 0.88 \mu \mathrm{M}$ in the serum iron level, respectively, whereas groups B and D exhibited increases of $1.57 \pm 0.69 \mu \mathrm{M}$ and $1.19 \pm 0.53 \mu \mathrm{M}$, respectively. There were significant differences in the changes in the serum iron level following intervention among the four groups, except between groups B and D.

Influences of different interventions on the treatment outcomes for persistent diarrhea. The four groups with persistent diarrhea were subject to routine treatment in conjunction with VA and/or $\mathrm{Zn}$ supplementation. After 5 days, the treatment efficacy was compared among the 127 children (Table 8). The total efficacy rates in the four groups were $63.64 \%, 90.32 \%, 93.33 \%$ and $54.55 \%$, respectively, with groups $\mathrm{B}$ and $\mathrm{C}$ exhibiting the best outcomes, followed by group A. The $\chi^{2}$ test revealed that the differences between groups $\mathrm{B}$ and $\mathrm{C}$ and group $\mathrm{D}$ were significant $(p<0.05)$, as were the differences between group A and groups B and $\mathrm{C}(p<0.05)$. However, there was no apparent difference 
Table 7. The changes of the serum iron before and after intervention

\begin{tabular}{ccccc}
\hline Group & Number & $\begin{array}{c}\text { Mean of Fe level } \\
(\mu \mathrm{M})\end{array}$ & $\begin{array}{c}\text { Mean of Fe level after 14 days } \\
\text { intervention }(\mu \mathrm{M})\end{array}$ & $\begin{array}{c}\text { Mean of average increase } \\
(\mu \mathrm{M})\end{array}$ \\
\hline A & 33 & $13.13 \pm 5.05$ & $12.02 \pm 4.33$ & $-1.11 \pm 1.14^{\star, t, \pm}$ \\
B & 31 & $13.13 \pm 7.97$ & $14.70 \pm 7.86$ & $1.57 \pm 0.69^{\S, \|}$ \\
C & 30 & $13.12 \pm 6.25$ & $12.82 \pm 5.85$ & $-0.30 \pm 0.88^{\natural}$ \\
D & 33 & $14.59 \pm 6.10$ & $15.78 \pm 6.10$ & $1.19 \pm 0.53$ \\
\hline
\end{tabular}

Results are expressed as mean \pm SD. *Compared with B group, $p<0.001 .{ }^{\dagger}$ Compared with $C$ group, $p<0.05$. ${ }^{\ddagger}$ Compared with $D$ group, $p<0.001$. ${ }^{\S}$ Compared with C group, $p<0.001$. "Compared with D group, $p>0.05$. "Compared with $D$ group, $p<0.001$.

Table 8. Comparison of the curative effect among the four groups after 5 days intervention

\begin{tabular}{|c|c|c|c|c|c|}
\hline \multirow{2}{*}{ Group } & \multirow{2}{*}{ Number } & \multicolumn{3}{|c|}{ The curative effect } & \multirow{2}{*}{$\begin{array}{c}\text { The total effective* } n \\
(\%)\end{array}$} \\
\hline & & Excellence $n(\%)$ & Effective $n(\%)$ & Invalid $n(\%)$ & \\
\hline A & 33 & $6.0(18.18)$ & $15.0(45.45)$ & $12.0(36.36)$ & $21.0(63.64)^{t, \neq}$ \\
\hline B & 31 & $8.0(25.81)$ & $20.0(64.52)$ & $3.0(9.68)$ & $28.0(90.32)^{\S}$ \\
\hline $\mathrm{C}$ & 30 & $7.0(23.33)$ & $21.0(70.0)$ & $2.0(6.67)$ & $28.0(93.33)^{\S}$ \\
\hline D & 33 & $4.0(12.12)$ & $14.0(42.42)$ & $15.0(45.45)$ & $18.0(54.55)$ \\
\hline
\end{tabular}

*The total effective $=$ Excellence + Effective. ${ }^{\dagger}$ Compared with $\mathrm{B}$ group, $p<0.05$. ${ }^{\ddagger}$ Compared with $\mathrm{C}$ group, $p<0.01 .{ }^{\mathrm{s}} \mathrm{Compared}$ with $\mathrm{D}$ group, $p<0.01$.

Table 9. Comparison of the curative effect among the four groups after 14 days intervention

\begin{tabular}{ccccc}
\hline \multirow{2}{*}{ Group } & \multirow{2}{*}{ Number } & \multicolumn{3}{c}{ The curative effect } \\
\cline { 3 - 5 } & & Excellence $n(\%)$ & The total effective* $n$ \\
$(\%)$
\end{tabular}

*The total effective $=$ Excellence + Effective. ${ }^{\dagger}$ Compared with $\mathrm{D}$ group, $p<0.05$. ${ }^{\ddagger}$ Compared with $\mathrm{D}$ group, $p<0.01 .{ }^{\S} \mathrm{Compared}$ with $\mathrm{D}$ group, $p<0.05$.

between groups $\mathrm{B}$ and $\mathrm{C}(p>0.05)$. These results indicate that short-term $\mathrm{Zn}$ and $\mathrm{VA}+\mathrm{Zn}$ supplementation can improve, to different degrees, the treatment outcomes of children with persistent diarrhea.

Table 9 summarises the treatment outcomes of the patients with persistent diarrhea. Twenty four, 26 and 25 patients in groups A, $\mathrm{B}$ and $\mathrm{C}$, respectively, exhibited improvements in their persistent diarrhea, representing superior efficacy to the improvement of the 14 patients in group $\mathrm{D}(p<0.05)$. The total effectiveness rates of groups A, B, and C were $93.94 \%, 96.77 \%$ and $96.67 \%$, respectively, and there were no significant differences among the three groups. In contrast, the three groups exhibited superior efficacy relative to group D $(72.73 \%)$, as revealed by the $\chi^{2}$ test $(p<0.05)$. These results indicate that when treating paediatric patients with persistent diarrhea using conventional approaches, simultaneous supplementation with VA, $\mathrm{Zn}$ or $\mathrm{VA}+\mathrm{Zn}$ can improve the therapeutic outcomes.

\section{Discussion}

Persistent diarrhea is a common paediatric disease among Chinese children, of which, the aetiologies are complex and numerous. In the present study, the top three causes of persistent diarrhea were FAG $(42 \%)$, secondary lactose intolerance $(17 \%)$ and AAD $(15 \%)$ in the 127 paediatric patients. However, enteric bacterial infection was only present in $5 \%$ of the 127 subjects with persistent diarrhea, where the primary pathogenic bacteria were Escherichia coli and Salmonella. In addition, there were 15 cases (11\%) with unclear causes of persistent diarrhea.
Persistent diarrhea is also a frequent cause of childhood malnutrition and growth retardation, which may be life-threatening. VA deficiency is ubiquitously present in developing countries and is associated with increased incidences of pneumonia and diarrhea. As such, enhanced VA supplementation has significantly decreased the hospitalisation rate of children with chronic diarrhea in Bangladeshi. ${ }^{(14)}$ Nevertheless, it remains unclear whether VA can assist treatment for persistent diarrhea.

Among the 127 children with persistent diarrhea examined in this study, there were 39 cases of low body weight and 41 cases of anaemia, yielding incidence rates of $30.71 \%$ and $32.28 \%$, respectively, which were both significantly higher than the national averages. ${ }^{(9-11)}$ In addition, $59.84 \%$ of the children exhibited various levels of hypoalbuminemia. Our results also revealed that the average serum VA level in the study sample was lower than that of healthy children under 5 years old in China (1.06 \pm $0.33 \mu \mathrm{M}){ }^{(12)}$ which is consistent with the results of Abrol. ${ }^{(15)}$ These findings indicated that low VA levels are commonly present in children with persistent diarrhea, possibly because protracted diarrhea leads to the malabsorption of nutrients that, in turn, results in anaemia, malnutrition, poor immune function and growth and development disorders.

In this study, we randomly divided the 127 subjects with persistent diarrhea into four groups, which did not differ with respect to age, gender, serum VA level or serum $\mathrm{Zn}$ and Fe level before the interventions. The children were provided with auxiliary treatment with VA (group A), Zn (group B) or VA + Zn (group C), with the $\mathrm{Zn}$ treatment serving as the positive control for auxiliary treatment and no intervention serving as the negative control 
(group D). ${ }^{(16)}$ Supplementation with VA and VA + Zn increased the serum VA levels and ameliorated anaemia. These results are consistent with our previous studies. ${ }^{(17-19)}$ Among these treatments, VA $+\mathrm{Zn}$ supplementation exhibited the most pronounced improvement effects on childhood anaemia, indicating that concurrent intervention with multiple micronutrients may result in synergistic modulation. ${ }^{(16,20-22)}$ Single $\mathrm{Zn}$ supplementation did not improve the serum VA level or anaemic status of the patients with persistent diarrhea; there were no significant differences between the $\mathrm{Zn}$ group and the control group. This finding indicates that $\mathrm{Zn}$ treatment cannot improve the VA nutritional level or anaemia of children with persistent diarrhea.

As is known, serum iron (SI) primarily reflects the stock status of liver iron. An increasing SI level results in an increase in the iron reserve, whereas a decreasing SI level indicates a decrease in the iron reserve. Either iron deficiency or increasing iron mobilisation can decrease the SI level. A study has revealed that VA improves the storage and utilisation of iron but has only a slight effect on the absorption of iron. ${ }^{(23)}$ Consistent with Chen's report, we also found that VA supplementation decreased the SI level and increased the $\mathrm{Hb}$ level of the paediatric patients with persistent diarrhea, indicating that VA could promote $\mathrm{Hb}$ synthesis by increasing iron mobilisation. ${ }^{(24)}$ Consequently, the decrease in SI level was associated with mobilisation of liver reserve iron and not an iron deficiency. $\mathrm{Zn}$, as an important trace element in human, widely exists in the body and is crucial in biological functions. Our study revealed that serum iron level increased following single $\mathrm{Zn}$ administration; however, there was no significant difference compared with the non-intervention group. Although recent studies have found that zinc supplementation decreases the serum iron concentration in healthy schoolchildren and non-anaemic women, short-term zinc intervention cannot significantly change the serum iron concentration. ${ }^{(20,21,25)}$ It was intriguing that the concurrent $\mathrm{VA}+\mathrm{Zn}$ supplementation slighly decreased the SI level of peadiatric patients. The reason was speculated to be a double effects in which VA promoted iron mobilisation but did not affect iron absorption; VA $+\mathrm{Zn}$ had the best treatment efficacy that increased serum iron level in a short period of time. ${ }^{(19)}$ Determining the exact mechanism requires further study.

A comparison of the treatment efficacy for persistent diarrhea performed after 5 days of treatment revealed that the total effectiveness rates of the $\mathrm{Zn}$ and VA $+\mathrm{Zn}$ supplementation were both significantly higher than those of the control group and single VA supplementation; in addition, there was no significant difference in treatment efficacy between the control group and single VA group or between the concurrent $\mathrm{VA}+\mathrm{Zn}$ group and the single $\mathrm{Zn}$ group. Nonetheless, as the treatment time extended, the VA supplementation resulted in a superior outcome relative to the control group, whereas the other three intervention treatments exhibited no significant differences, which is consistent with a study by Walser. ${ }^{(22)}$

VA is increasingly recognised to counteract infection and promote epithelial barrier function and intestinal mucosal immunity. Studies have indicated that retinoic acid (RA), an important intermediate metabolite of VA, can induce T and B cells to express homing receptors $\alpha 4 \beta 7$ and CCR9, which thereby regulates mucosal immune responses. ${ }^{(26,27)}$ Our preliminary experiments also revealed that VA deficiency during pregnancy or in early life can decrease intestinal IgA secretion and influences the numbers of B cells, dendritic cells and T cells in gut-associated lymphoid tissues. ${ }^{(28)}$ Meta-analyses indicate that $\mathrm{Zn}$ supplementation can decrease the duration and severity of persistent diarrhea. ${ }^{(29,30)}$ Likewise, Rahman reported that concurrent VA $+\mathrm{Zn}$ supplementation can significantly reduce the incidence of persistent diarrhea. ${ }^{(16)}$ Therefore, we investigated VA supplementation and used a $\mathrm{Zn}$ auxiliary treatment as a positive control, thereby generating three interventional approaches. A comparison revealed that $\mathrm{Zn}$ signifi- cantly enhanced the treatment efficiency in patients with persistent diarrhea, whereas VA supplementation promoted treatment efficacy, enhanced the VA level and improved the anaemic status of the children. These results therefore indicate that concurrent supplementation of VA and $\mathrm{Zn}$ is the optimal auxiliary treatment for persistent diarrhea and can break the vicious cycle of "diarrhea-malnutrition-diarrhea" and protect children from growth retardation.

Gut microbiota imbalances damage the intestinal barrier and may contribute to diarrhea-predominant irritable bowel syndrome, inflammatory bowel disease and necrotising enterocolitis. ${ }^{(31)}$ Patients with diarrhea-predominant irritable bowel syndrome exhibit a reduced number of beneficial microbiota, elevated numbers of harmful microorganisms and decreased intestinal microbial diversity. ${ }^{(32,33)}$ Furthermore, gut flora imbalances and damage to the intestinal barrier are crucial pathogenic factors. ${ }^{(34)}$ This study also revealed that subjects with dysbiosis represented $94.49 \%$ of the persistent diarrhea cohort, suggesting a close association between persistent diarrhea and gut dysbiosis. Jejunum, ileum and colon tissues deficient in VA exhibit reductions in Lactobacillus spp. of $62 \%, 82 \%$ and $86 \%$, respectively, as well as a striking elevation of Escherichia coli strains. ${ }^{(35)}$ Our study demonstrated that subjects with simultaneous VAD and dysbiosis accounted for $78.74 \%$ of the study cohort and that the proportion of severe dysbiosis subjects in the VAD group was markedly higher than that of the VAN group, suggesting an intricate relationship between VA nutrition status and dysbiosis in children with persistent diarrhea. A future focus of our group will be to elucidate whether VA auxiliary treatment can ameliorate gut dysbiosis in children with persistent diarrhea and how the VA nutritional level regulates intestinal microbiota at the molecular level.

In summary, our study reveals that children with persistent diarrhea exhibit a plethora of prominent nutrition problems, including low body weight, anaemia, VA deficiency and hypoproteinemia. Our data also support a close association between VA nutrition level and faecal dysbiosis in paediatric patients. In addition, single VA supplementation, single $\mathrm{Zn}$ supplementation, and concurrent VA + Zn supplementation can significantly boost the therapeutic efficacy of treatment for persistent diarrhea and decrease the disease duration. However, concurrent $\mathrm{VA}+\mathrm{Zn}$ supplementation generates the best outcome by increasing the overall treatment effectiveness rate for persistent diarrhea as well as by improving the nutritional status of paediatric patients.

\section{Acknowledgments}

This work was supported by National Nature Science Foundation of China (grant number 81070286, 81470799) and Science \& Technology Project of Yuzhong District of Chongqing (2012). We would like to thank all the paediatric patients enrolled in this study and their guardians.

\section{Abbreviations}

$\begin{array}{ll}\text { AAD } & \text { antibiotic-associated diarrhea } \\ \text { Alb } & \text { albumin } \\ \text { FAG } & \text { food allergic gastrointestinal disease } \\ \text { SI } & \text { serum iron } \\ \text { VA } & \text { vitamin A } \\ \text { VAD } & \text { vitamin A deficiency } \\ \text { VAN } & \text { vitamin A normal }\end{array}$

\section{Conflict of Interest}

No potential conflicts of interest were disclosed. 


\section{References}

1 Andrade JA, Fagundes-Neto U. Persistent diarrhea: still an important challenge for the pediatrician. J Pediatr (Rio J) 2011; 87: 199-205.

2 Fagundes-Neto U. Persistent diarrhea: still a serious public health problem in developing countries. Curr Gastroenterol Rep 2013; 15: 345.

3 Cao J, Wei X, Tang X, et al. Effects of egg and vitamin A supplementation on hemoglobin, retinol status and physical growth levels of primary and middle school students in Chongqing, China. Asia Pac J Clin Nutr 2013; 22: 214-221.

4 Qiu XG, Chen X, Yang SW, Huang YC, Dai LQ. Effect of vitamin A deficiency of children on infectious diseases. Maternal \& Child Health Care of China 2009; 24: 2684-2686.

5 Umamaheswari B, Biswal N, Adhisivam B, Parija SC, Srinivasan S. Persistent diarrhea: risk factors and outcome. Indian J Pediatr 2010; 77: 885-888.

6 Pawlowski SW, Warren CA, Guerrant R. Diagnosis and treatment of acute or persistent diarrhea. Gastroenterology 2009; 136: 1874-1886.

7 Fang HS, Wei CY. Diagnosis and treatment of diarrhea disease in China. Chinese Journal of Practical Pediarics 1998; 13: 381-384.

8 Zhang XR. Intestinal flora fecal smears atlas. Beijing: People's Medical Publishing House, 2000.

9 Yang XG, Wang ZH, He YN, Yu WT, Hu YS, Zhai FY. Trends and prevalence of malnutrition among Chinese children under five years old. Acta Nutrimenta Sinica 2005; 27: 185-188.

$10 \mathrm{Li}$ LM, Rao KQ, Kong LZ, et al. A description on the Chinese national nutrition and health survey in 2002. Zhonghua Liu Xing Bing Xue Za Zhi 2005; 26: 478-484.

11 Piao JH, Lai JQ, Yin SA, Xu J, Xu QM, Yang XG. Study on the anemia status of Chinese population. Acta Nutrimenta Sinica 2005; 04: 268-271+275.

12 Lin L, Liu Y, Ma G, et al. Survey on vitamin A deficiency in children under6-years in China. Zhonghua Yu Fang Yi Zue Za Zhi 2002; 36: 315-319.

13 All-China WsF. Nutrition and Health Status of Chinese Residents. 2005.

14 Das SK, Faruque AS, Chisti MJ, Malek MA, Salam MA, Sack DA. Changing trend of persistent diarrhoea in young children over two decades: observations from a large diarrhoeal disease hospital in Bangladesh. Acta Paediatr 2012; 101: e452-e457.

15 Abrol P, Mehta U, Lal H. Vitamin a status in children with diarrhoea. Indian J Clin Biochem 2002; 17: 64-68.

16 Rahman MM, Vermund SH, Wahed MA, Fuchs GJ, Baqui AH, Alvarez JO. Simultaneous zinc and vitamin A supplementation in Bangladeshi children: randomised double blind controlled trial. BMJ 2001; 323: 314-318.

17 Zhang X, Chen K, Qu P, Liu YX, Li TY. Effect of biscuits fortified with different doses of vitamin A on indices of vitamin A status, haemoglobin and physical growth levels of pre-school children in Chongqing. Public Health Nutr 2010; 13: 1462-1471.

18 Liu YF, Chen L, Gong M, et al. Effects of vitamin A combined with other micronutriens on nutritional status of 3-6 years old children. Academic Journal of Second Military Medical University 2013; 33: 828-834.

19 Chen K, Liu YF, Chen L, et al. Effects of vitamin A, vitamin A plus iron and multiple micronutrient-fortified seasoning powder on iron metabolic homeostasis. Chin J Pediatr 2011; 49: 926-932.

20 de Brito NJ, Rocha ÉD, de Araújo Silva A, et al. Oral zinc supplementation decreases the serum iron concentration in healthy schoolchildren: a pilot study. Nutrients 2014; 6: 3460-3473.

21 Zaka-ur-Rab Z, Ahmad SM, Naim M, Alam S, Adnan M. Effect of short term zinc supplementation on iron status of children with acute diarrhea. Indian J Pediatr 2015; 82: 421-426.

22 Walser BL, Lima AA, Guerrant RL. Effects of high-dose oral vitamin A on diarrheal episodes among children with persistent diarrhea in a northeast Brazilian community. Am J Trop Med Hyg 1996; 54: 582-585.

23 Milman N. Anemia--still a major health problem in many parts of the world! Ann Hematol 2011; 90: 369-377.

24 Chen K, Zhang L, Luo HY, Wang J, Li Q, Mao M. No enhancing effect of vitamin A administration on iron absorption or body total iron content in preschool children from Chengdu, China. J Nutr Sci Vitaminol (Tokyo) 2014; 60: $223-230$.

25 Zaman K, McArthur JO, Abboud MN, et al. Iron supplementation decreases plasma zinc but has no effect on plasma fatty acids in non-anemic women. Nutr Res 2013; 33: 272-278.

26 Mora JR, Iwata M, Eksteen B, et al. Generation of gut-homing IgA-secreting B cells by intestinal dendritic cells. Science 2006; 314: 1157-1160.

27 Iwata M, Hirakiyama A, Eshima Y, Kagechika H, Kato C, Song SY. Retinoic acid imprints gut-homing specificity on T cells. Immunity 2004; 21: 527-538.

28 Liu X, Li Y, Wang Y, et al. Gestational vitamin A deficiency reduces the intestinal immune response by decreasing the number of immune cells in rat offspring. Nutrition 2014; 30: 350-357.

29 Lazzerini M, Ronfani L. Oral zinc for treating diarrhoea in children Cochrane Database Syst Rev 2012; 6: CD005436.

30 Lukacik M, Thomas RL, Aranda JV. A meta-analysis of the effects of oral zinc in the treatment of acute and persistent diarrhea. Pediatrics 2008; 121: 326-336.

31 Weber TK, Polanco I. Gastrointestinal microbiota and some children diseases: a review. Gastroenterol Res Pract 2012; 2012: 676585.

32 Carroll IM, Ringel-Kulka T, Siddle JP, Ringel Y. Alterations in composition and diversity of the intestinal microbiota in patients with diarrhea-predominant irritable bowel syndrome. Neurogastroenterol Motil 2012; 24: 521-530, e248.

33 Ohman L, Simrén M. Intestinal microbiota and its role in irritable bowel syndrome (IBS). Curr Gastroenterol Rep 2013; 15: 323.

34 Carroll IM, Ringel-Kulka T, Keku TO, et al. Molecular analysis of the luminal- and mucosal-associated intestinal microbiota in diarrhea-predominant irritable bowel syndrome. Am J Physiol Gastrointest Liver Physiol 2011; 301: G799-G807.

35 Amit-Romach E, Uni Z, Cheled S, Berkovich Z, Reifen R. Bacterial population and innate immunity-related genes in rat gastrointestinal tract are altered by vitamin A-deficient diet. J Nutr Biochem 2009; 20: 70-77. 